\title{
Effects of Target Expansion on Selection Performance in Older Computer Users
}

\author{
FAUSTINA HWANG and NIC HOLLINWORTH, University of Reading \\ NITIN WILLIAMS, University of Cambridge
}

\begin{abstract}
Point and click interactions using a mouse are an integral part of computer use for current desktop systems. Compared with younger users though, older adults experience greater difficulties performing cursor positioning tasks, and this can present limitations to using a computer easily and effectively. Target expansion is a technique for improving pointing performance where the target grows dynamically as the cursor approaches. This has the advantage that targets conserve screen real estate in their unexpanded state, yet can still provide the benefits of a larger area to click on. This article presents two studies of target expansion with older and younger participants, involving multidirectional point-select tasks with a computer mouse. Study 1 compares static versus expanding targets, and Study 2 compares static targets with three alternative techniques for expansion. Results show that expansion can improve times by up to $14 \%$, and reduce error rates by up to $50 \%$. Additionally, expanding targets are beneficial even when the expansion happens late in the movement, that is, after the cursor has reached the expanded target area or even after it has reached the original target area. The participants' subjective feedback on the target expansion are generally favorable, and this lends further support for the technique.
\end{abstract}

Categories and Subject Descriptors: H.5.2 [Information Interfaces and Presentation]: User Interfaces-Graphical user interfaces (GUI); Interaction styles

General Terms: Human Factors, Experimentation, Performance

Additional Key Words and Phrases: Older adults, expanding targets, expansion, computer mouse, target selection, point and click

\section{ACM Reference Format:}

Hwang, F., Hollinworth, N., and Williams, N. 2013. Effects of target expansion on selection performance in older computer users. ACM Trans. Access. Comput. 5, 1, Article 1 (September 2013), 26 pages.

DOI : http://dx.doi.org/10.1145/2514848

\section{INTRODUCTION}

Point and click interactions using a computer mouse to select targets on a screen are an integral part of computer use for current desktop systems. Compared with younger users though, older adults experience greater difficulties performing cursor positioning tasks [Chaparro et al. 1999; Smith et al. 1999]. Older adults take longer to complete selections, and need to spend a greater proportion of the overall movement time homing-in on a target. They also make a higher number of corrective submovements in order to select a target to the same level of accuracy as younger users, particularly

\footnotetext{
This work was supported by the Strategic Promotion of Ageing Research Capacity (SPARC) initiative, funded jointly by the UK Engineering and Physical Sciences Research Council (EPSRC) and Biotechnology and Biological Sciences Research Council (BBSRC).

Authors' addresses: F. Hwang (corresponding author) and N. Hollinworth, School of Systems Engineering, University of Reading; email: f.hwang@reading.ac.uk; N. Williams, MRC Cognition and Brain Sciences Unit, University of Cambridge.

Permission to make digital or hard copies of part or all of this work for personal or classroom use is granted without fee provided that copies are not made or distributed for profit or commercial advantage and that copies bear this notice and the full citation on the first page. Copyrights for third-party components of this work must be honored. For all other uses, contact the Owner/Author.
}

2013 Copyright is held by the author/owner(s).

1936-7228/2013/09-ART1

DOI : http://dx.doi.org/10.1145/2514848 
for smaller targets [Ketcham et al. 2002, 2004; Walker et al. 1997]. Thus, for the older population, difficulties with cursor control can present a significant limitation to using a computer easily and effectively [Sandfeld and Jensen 2005; Sutter and Müsseler 2007].

In the human-computer interaction research community, there has been interest in target expansion as a method of improving performance in target acquisition [Cockburn and Brock 2006; McGuffin and Balakrishnan 2002, 2005; Zhai et al. 2003]. With this technique, the target grows in size as the cursor approaches, with the advantage that the targets conserve screen real estate in their unexpanded state, yet can still provide the benefits of having a larger area to click on. These studies reported improvements in selection times even when the expansion occurred after the cursor was $90 \%$ of the initial distance to the target [McGuffin and Balakrishnan 2005]. Improved performance has also been reported when using visual expansion alone, with unaltered motor space [Cockburn and Brock 2006]. Hence target expansion would appear promising in its potential to improve target selection, and already features in some commercial systems.

These earlier studies, however, were mainly conducted with younger participants. Yet, age-related changes in cognitive, perceptual and motor capabilities affect how older people interact with computers [Czaja and Lee 2003; Worden et al. 1997], and so the responses of younger users to novel interaction techniques may not be reflective of how older users will respond to the same techniques. Older users may benefit more from some interaction methods than younger users (as was the case with sticky icons and area cursors [Worden et al. 1997]), but equally, may find some techniques less helpful or possibly detrimental (as was found with proxy targets [Hwang et al. 2008]). For older users, expanding targets may help reduce the time required for homing-in on a target by reducing the need for fine motor control. However, older people also take longer to process incoming information and typically require more time to respond to changes [Czaja and Lee 2003], which may hamper the ability to take advantage of an expanding target. There is also the concern that older adults may find expanding targets distracting. Thus, to understand how older adults' performance is affected by target expansion, it is important to study this technique with the older population directly.

To our knowledge, there have been only two reported studies of target expansion with older computer users. Bohan and Scarlett's [2003] was a preliminary experiment with eight younger (mean age $=20$ years) and eight older (mean age $=81$ years) participants, performing 20 trials of the same point and click task in each of five different target conditions - two sizes of static targets (small and large), and three conditions where targets expanded from the small to the large size but at different expansion points, that is, at $10 \%, 50 \%$, and $90 \%$ of the distance to the target. Their implementation of the expansion differed from the studies by other researchers, in that once the cursor had crossed the expansion point, rather than having the expansion occur at a set rate, the size of the target was related to the cursor's distance from the target, increasing or decreasing in size as the cursor moved toward or away from the target's center. They found that when expansion occurred late in the movement, neither group benefited from the target expansion. However, when the expansion occurred early on in the movement, both older and younger users were able to take advantage of the larger final target size. This indicates that older adults could potentially benefit from target expansion, but also raises further questions about the conditions under which the expansion is helpful.

A more recent paper by Lee et al. [2012] presents a comparative evaluation of different expansion designs in two-dimensional target configurations. Their study involved 14 younger adults under 60 years of age (aged 24 to 49) and 14 older adults over 
60 years of age (aged 61 to 76). In contrast with Bohan and Scarlett's [2003] singletarget experiment, Lee et al.'s [2012] experiment presented 114 icons that were densely arranged into 6 groups across the screen, and the task was to select one icon from within the 2D arrangement. Each participant completed 30 trials in each of 8 conditions which were a combination of four different expansion areas (i.e., what was expanded on the screen - ranging from nothing/static target to expansion of an entire group of icons) and two expansion techniques (one where an expanded icon or group of icons would occlude adjacent targets, and the other where they would push neighbouring targets aside, hence changing their location on the screen). In contrast with other studies, they found no improvement in time with target expansion over a static condition, and also found that in some conditions the expansion had a detrimental effect on speed. However, in some conditions, error rates were reduced with expansion, and participants also reported a favorable subjective response to the expansion. As with Bohan and Scarlett's [2003] study, this study provides indications of the benefits of target expansion for older adults, but also highlights that, under certain conditions, target expansion can be ineffective or even detrimental.

These two studies are very different in their experimental design. Bohan and Scarlett [2003] used a simple selection task and an unusual implementation of the expansion. Lee et al. [2012] used a more common implementations of expansion, but although the aim of investigating more realistic scenarios is undoubtedly important, the combination of dense 2D arrangements of targets with eight different expansion implementations has potentially obscured a more fundamental understanding of how older adults respond to target expansion. The contrasting results from these two studies suggests that further research in this area is warranted. A better understanding of how older adults respond to expanding targets may help to inform designers as to whether expanding targets should be incorporated into interfaces for older people, and if this is the case, which techniques are suitable and how they should be implemented. The current work complements the work of Bohan and Scarlett [2003] and Lee et al. [2012], and adds to the body of empirical data on the effects of target expansion on selection performance of older adults.

This article presents two studies of target expansion, both comparing older and younger participants in point-select tasks with a computer mouse. Study 1 compares two conditions, static versus expanding targets, where the target expansion method is based on that used in other studies [Cockburn and Brock 2006; McGuffin and Balakrishnan 2002, 2005; Zhai et al. 2003]. In contrast with Bohan and Scarlett [2003] where the size of the target was inversely proportional to the distance of the cursor from the target's center and hence was changing with cursor movement, with the technique used here, the target stays at its fully-expanded size and only shrinks back to the original size when the cursor is greater than $50 \%$ of the initial distance from the target. The results from Study 1 show that target expansion can improve speed and accuracy for both older and younger adults. Hence, Study 2 builds on this and examines the potential of expanding targets further by comparing static targets with three alternative techniques for expansion; as designs for target expansion can vary, it is useful to conduct side-by-side evaluations of different designs in order to better understand how performance compares across them [Lee et al. 2012].

\section{RELATED WORK}

\subsection{Older Adults and Computer Pointing Devices}

As noted by Wood et al. [2005], "one of the first challenges seniors encounter with computers involves navigating the use of the input device." In a course on computers for beginners aged over 55, Dickinson et al. [2010] reported mouse use as an initial 
barrier for the students. Although in most cases, students were able to learn relatively quickly how to use the mouse to a sufficient standard to perform basic computing tasks, certain mouse tasks such as double-clicking and dragging to select text were a regular "irritation" for some and a "significant barrier" for others, and, in two cases, persistent difficulties with using the mouse rendered standard computer technology effectively unusable. Hence, the use of computer pointing devices by older adults has been a subject of interest in the research community.

In terms of target selection by using a mouse, studies have shown that older adults have more difficulties in selecting targets than younger users [Czaja and Lee 2003; Smith et al. 1999]. Older adults take longer and make more errors, particularly with smaller and more distant targets [Walker et al. 1997; Worden et al. 1997]. This has been attributed to age-related changes in motor and perceptual capabilities [Walker et al. 1997], and differences in pointing behaviors such as lower peak velocities, increased deceleration times, more frequent pauses during movements, and more target reentries and slip-offs [Keates and Trewin 2005; Smith et al. 1999].

Research with older adults has also extended to pointing devices other than the computer mouse, for instance, pen-based input on tablets and handheld computers [Hourcade and Berkel 2008; Moffatt and McGrenere 2007, 2009], touchscreens on touch-panel interfaces, mobile phones, and surfaces [Murata and Iwase 2005; Nicolau and Jorge 2012; Piper et al. 2010], and other input devices (e.g., large rollerballs and touchpads) [Taveira and Choi 2009; Wood et al. 2005]. These studies aim to better understand and to characterize the nature of older adults' interactions with pointing devices as a basis for informing the design of novel input devices, software interfaces, and assistive techniques. In many cases, older adults are compared with younger ones, and the results highlight important differences between the age groups and identify challenges unique to the older population, hence highlighting the importance of conducting studies explicitly with older adults.

\subsection{Techniques to Assist Target Selection for Older Adults}

Improving target selection with a computer mouse has been the subject of much research in the human-computer interaction community. Techniques proposed have included, for example, resizing the activation area of the cursor (e.g., bubble cursors [Grossman and Balakrishnan 2005]); resizing the target (e.g., target expansion [McGuffin and Balakrishnan 2005]); various methods of mapping motor movement to cursor movement (e.g., semantic pointing [Blanch et al. 2004]; object pointing [Guiard et al. 2004]); force fields [Ahlström et al. 2006]); using multimodal feedback (e.g., audio and haptics [Cockburn and Brewster 2005]); and multimodal input (e.g., rake cursor [Blanch and Ortega 2009]; MAGIC [Zhai et al. 1999]); and using multiple cursors (e.g., Ninja cursors [Kobayashi and Igarashi 2008]; satellite cursors [Yu et al. 2010]). This section focuses, though, on studies with an explicit interest in older adults.

Research on techniques to improve target selection for older adults covers different aspects of the task - clicking on the target, moving to the target, and finding the cursor on the screen in order to begin moving to the target. Worden et al. [1997] proposed area cursors and sticky icons. Area cursors have a larger than normal activation area, and sticky icons decrease the cursor's control-display gain as the cursor passes over a target [Worden et al. 1997]. Both of these techniques were found to decrease target selection times for older adults. Steady Clicks [Trewin et al. 2006] is an assistive technique designed to reduce slips, that is, click errors where the cursor slips off a target before the mouse button is released, and accidental clicks, that is, clicks that occur en-route to the target or that occur while trying to press a different button. Slips are prevented by freezing the cursor position during a click, and accidental clicks are prevented by suppressing clicks made while the cursor is moving at a high speed and 
by ignoring overlapping button presses. In a study with 11 motor-impaired people, including three who were 60+, they found that Steady Clicks reduced the number of click attempts required to select a target successfully. PointAssist [Hourcade et al. 2008] is a technique designed to assist target selection by automatically detecting when a user is experiencing difficulty (as indicated by a series of slow, short submovements in the cursor behavior), and triggering a precision mode that reduces the speed of the cursor by half. In a study with twenty older adults (ages 66-88), PointAssist did not significantly improve times, but for some participants, the technique did improve accuracy. Proxy targets aim to reduce the distance that the cursor must travel, and hence the movement time by bringing the target closer to the cursor once a pointing movement has started. In a controlled experiment with a "laboratory" implementation of proxy targets, for younger users, proxies did not provide any benefits over direct selection, while for older users, times were increased with proxy targets [Hwang et al. 2008]. The authors discuss the possibility that the benefits of proxy targets may only become apparent when interacting with targets over distances larger than those examined in their experiment, and note that for any further research in this area, it was encouraging that older adults did not appear to have any difficulty adapting to the proxy targets.

The work by Hollinworth and Hwang [2011] is aimed at helping people to find the cursor on the screen prior to initiating the pointing movement. Older adults often report "losing" the cursor on the screen, which can present a barrier to effective computer use. In this study, a touch sensor was embedded inside a standard computer mouse, so that when the mouse was released and subsequently touched, the mouse was automatically centered to the screen. In a point and click task for which the starting point of the cursor was unknown, both older and younger users were fastest with this technique, compared with more traditional methods of locating a cursor on the screen (i.e., shaking the mouse from side to side, and the built-in Windows mouse sonar). For older adults, target selection times were reduced by up to two seconds.

There has also been research on techniques to support target selection with pen input on tablets. Moffatt and McGrenere [2010] developed Steadied Bubbles, which combined a circular bubble cursor that would grow and shrink to capture the nearest target while the pen-tip was within the hover range of the display, with the steady click technique that would freeze the cursor in location and size once the pen touched the screen. In a study with 12 younger and 12 older adults, they found that Steadied Bubbles could lower the incidence of users missing the target and slipping off the target. Although both age groups benefited, the effect was larger for the older adults.

Compared with the wider HCI community, research on target selection for older adults has been less extensively investigated. Existing work includes examples where techniques have benefited older adults more than younger adults, as well as where they had a detrimental effect on performance for older users. There is much scope for further research in this area, and the results from previous studies illustrate the importance of investigating techniques directly with older adults.

\section{STUDY 1}

Study 1 investigates how performance for static targets compares with that for expanding targets for both older and younger adults.

\subsection{Participants}

Ten older adults and ten younger adults volunteered to participate in the study. The younger adults (mean age $=24.2$ years, range $=20$ to 29 years, 4 female, all righthanded) were students and researchers from the university. All were daily computer users. The older adults (mean age $=71.7$ years, range $=62$ to 83 years, 6 female, 


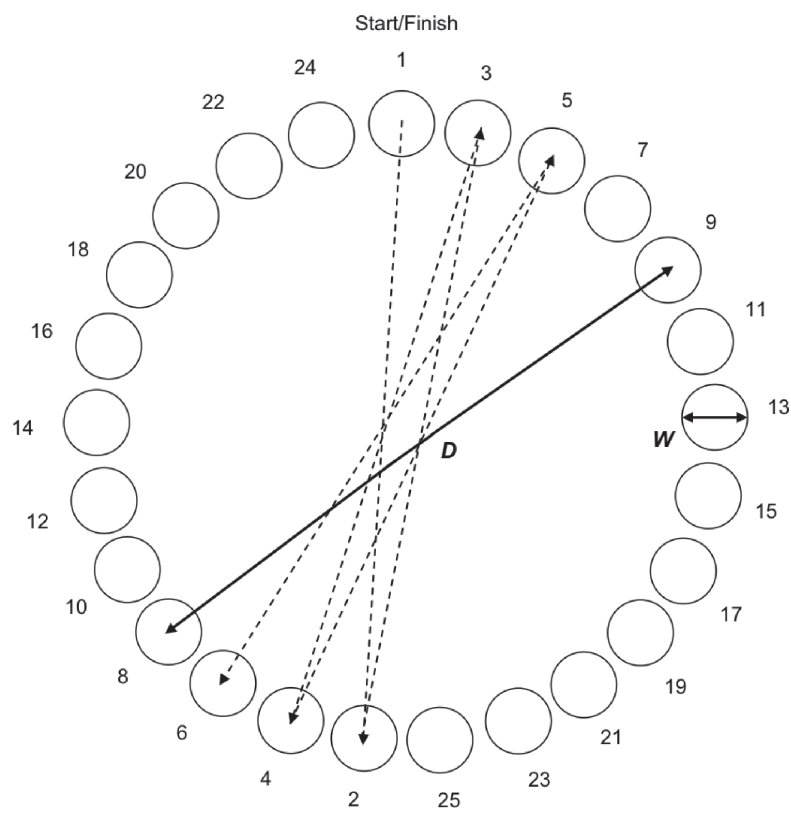

Fig. 1. Multidirectional pointing task. Arrows indicate the sequence of target selections. D and W indicate the target distance and width, respectively.

9 right-handed) were contacted through an IT training program for older people as well as through the university. All had some prior computer experience, with eight who reported use of computers at least once a week, but the level of computer experience was generally lower than that for the younger users. All participants reported normal or corrected vision, and none had any motor difficulties that restricted their use of computers.

\subsection{Apparatus}

The study was conducted on a Toshiba laptop (Satellite Pro A120) with a 15.4 inch widescreen, 1280 x 800 TFT display, running Windows XP Pro. An optical computer mouse was used to perform selection tasks.

\subsection{Tasks}

Study participants performed a serial, multidirectional selection task, based on the procedure recommended by the ISO 9241-9 standard [ISO 9241, 2000; Soukoreff and MacKenzie 2004]. The targets to be selected were circular, and equally spaced around the circumference of a larger circle, as illustrated in Figure 1. The outlines of all potential targets were visible on the screen throughout, and each target to be selected was filled in with red. Participants were asked to select targets as quickly and accurately as possible. Participants were also asked to leave errors uncorrected so that each trial ended with a click of the left mouse button, whether or not the click occurred inside the target. No feedback was given as to whether or not each selection was successful. The next trial started as soon as the user clicked in the previous trial.

Target selections proceeded in sequences of 25 trials, beginning with the topmost target, and with each subsequent selection on the target that was diametrically opposite. This sequence continued clockwise around the circle and ended when the topmost target had been selected again. At the end of each sequence, participants 
were given feedback about their average selection time and their error rate for that sequence. Participants were also encouraged to rest between sequences. After the selection tasks, participants were asked to complete a questionnaire, rating their perceived ease, speed, and accuracy of selecting expanding targets in comparison with selecting static targets. Participants were also asked to indicate their preference for the target type (expanding or static) and to rate their level of agreement with three statements pertaining to different aspects of the target expansion: visual appeal, degree to which the expansion was distracting, and degree to which the expansion was annoying. Responses for each item on the questionnaire used a 7-point Likert scale with an additional option to select "Don't know".

\subsection{Target Types}

Participants completed this task with two different target types - static and expanding. With static targets, the width of the target to be selected remained constant throughout a trial. With expanding targets, the target to be selected in each trial expanded to twice its original width when the cursor came within $50 \%$ of the initial distance to the target, that is, at an expansion point of $\mathrm{P}=0.5$ [McGuffin and Balakrishnan 2005]. Targets expanded over a 100ms period, as in McGuffin and Balakrishnan [2002], to give a transition that is rapid but not visually jarring. If the cursor subsequently moved farther away from the target, crossing back over this $\mathrm{P}=0.5$ threshold, the target instantly reverted back to its initial size.

\subsection{Design}

The study had a $2 \times 2 \times 11$ design with age (younger, older) as a between-subjects factor, and target type (static, expanding) and index of difficulty (ID) as within-subject factors. Selections were carried out in sequences of 25 trials, where the target type and the index of difficulty were held constant for the sequence. The $11 \mathrm{D} / \mathrm{W}$ combinations were, in pixels, 330/40, 270/32, 330/20, 270/16, 270/10, 330/12, 660/12, 600/10, 600/6, $660 / 6$, and $660 / 4$, giving IDs ranging from 3.21 to 7.38 bits. The 22 sequences (2 target types $\mathrm{x} 11 \mathrm{D} / \mathrm{W}$ combinations) were presented in random order.

At the start of the study, participants were given a brief demonstration of the task, followed by two practice sequences for each of the two target types. Data from these practice trials were not used in the analysis.

\subsection{Results}

Movement times (i.e., the time elapsed from the start of the trial to the mouse click) and error rates (i.e., percentage of trials where the user clicked outside the target) were analyzed with mixed design ANOVAs, with target type and ID as within-subject variables and age as a between-subject variable. The ID for an expanding target was calculated using the initial, unexpanded width.

For each participant, trials with movement times greater than three standard deviations from the mean for each condition were considered as outliers [Soukoreff and MacKenzie 2004] and removed. A visual inspection of the data also showed a small number of trials that were completed in less than $300 \mathrm{~ms}$. These appeared to be the result of an inadvertent selection, and were also removed. In total, the number of data points removed was less than $1 \%$ of the total number of data points. For each user, the mean of the remaining trials for each condition was then calculated and used in the analysis and the accompanying figures and tables.

3.6.1. Movement Times. Mean movement times for the two age groups and the two conditions are summarized in Table I and illustrated in Figure 2. The ANOVA showed a significant main effect of age $\left(\mathrm{F}_{1,18}=13.19, \mathrm{p}=0.002\right)$; the overall mean movement 
Table I. Mean Movement Times in Seconds (with standard errors shown in parentheses)

\begin{tabular}{|l|c|c|c|}
\hline & Older & Younger & Overall \\
\hline Static & $2.33 \mathrm{~s}(0.32)$ & $1.11 \mathrm{~s}(0.05)$ & $1.72 \mathrm{~s}(0.16)$ \\
\hline Expanding & $2.07 \mathrm{~s}(0.33)$ & $0.92 \mathrm{~s}(0.36)$ & $1.50 \mathrm{~s}(0.17)$ \\
\hline Overall & $2.2 \mathrm{~s}(0.32)$ & $1.0 \mathrm{~s}(0.04)$ & \\
\hline
\end{tabular}

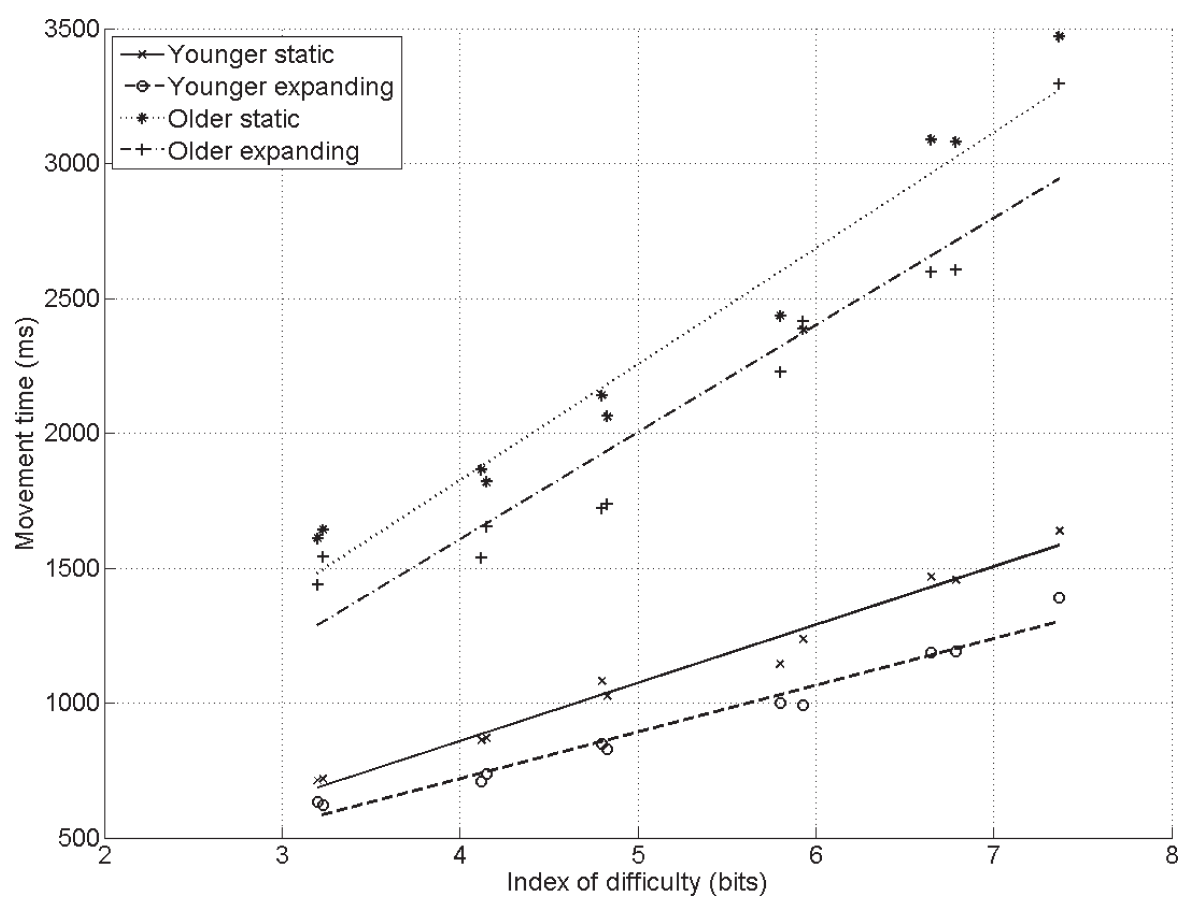

Fig. 2. Mean movement times versus ID for static and expanding targets, and older and younger users.

time for the older adults was more than two times longer than for the younger group (2.2s compared with $1.0 \mathrm{~s})$. A significant main effect of target type was also observed $\left(\mathrm{F}_{1,18}=49.01, \mathrm{p}<0.001\right)$, with participants performing faster with expanding targets than with static targets (overall mean of $1.5 \mathrm{~s}$ compared with $1.7 \mathrm{~s}$, an improvement of $13 \%$ with the expansion). The interaction between target type and age was not significant $\left(\mathrm{F}_{1,18}=1.15, \mathrm{p}=0.299\right)$. Separate analyses of movement times for older and younger participants showed a significant main effect of target type in both cases $\left(\mathrm{F}_{1,9}=12.77, \mathrm{p}=0.006\right.$ for younger participants, $\mathrm{F}_{1,9}=57.12, \mathrm{p}<0.001$ for older participants); for both age groups, performance was significantly improved with target expansion.

There was a significant main effect of $I D\left(\mathrm{~F}_{10,180}=78.94, \mathrm{p}<0.001\right)$, and a significant interaction between $I D$ and age $\left(\mathrm{F}_{10,180}=11.08, \mathrm{p}<0.001\right)$, with older adults appearing to be more affected by increasing task difficulty than younger users (Figure 2). There was also a significant interaction between $I D$ and target type $\left(\mathrm{F}_{10,180}=2.65\right.$, $\mathrm{p}=0.005$ ); Figure 2 suggests that this may be due in part to a very slight increase in the benefit that was gained from target expansion as the ID increased.

3.6.2. Error Rates. An error was defined to occur when the user clicked outside the target. For each user, the figure used in the ANOVA was the percentage of trials in each 
Table II. Mean Error Rates (with standard errors shown in parentheses)

\begin{tabular}{|l|l|c|r|}
\hline & Older & Younger & \multicolumn{1}{l|}{ Overall } \\
\hline Static & $28.98 \%(4.98 \%)$ & $11.46 \%(3.13 \%)$ & $20.2 \%(2.94 \%)$ \\
\hline Expanding & $15.49 \%(4.07 \%)$ & $3.64 \%(1.34 \%)$ & $9.6 \%(2.14 \%)$ \\
\hline Overall & $22.2 \%(4.46 \%)$ & $7.5 \%(2.15 \%)$ & \\
\hline
\end{tabular}

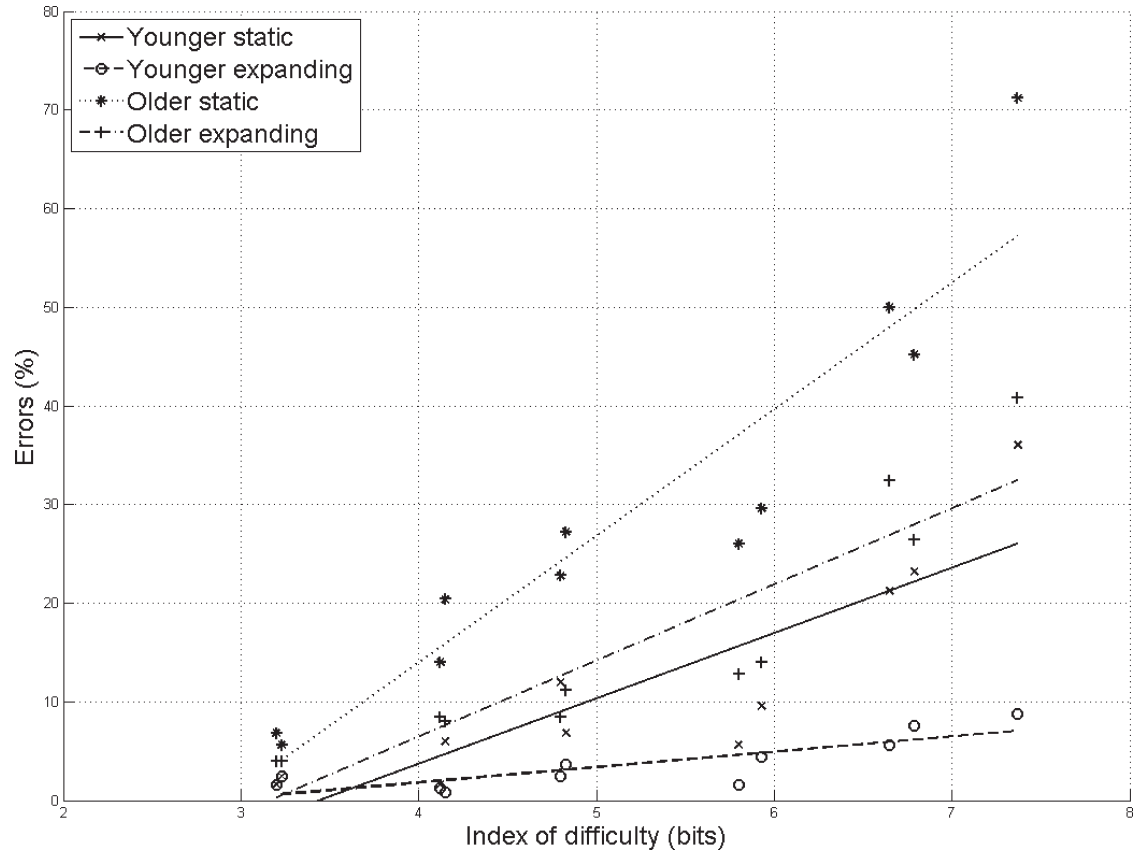

Fig. 3. Mean error rates versus ID for static and expanding targets, and older and younger users. The lines have been included to indicate the general trend of the data, and are not intended to suggest any theoretical linear relationship between error rates and ID.

condition that resulted in an error. Table II and Figure 3 show the mean error rates across participants, for both older and younger users and for static and expanding targets.

There was a significant main effect of age $\left(\mathrm{F}_{1,18}=8.82, \mathrm{p}=0.008\right)$, with the older users making more errors than the younger users (an overall mean error rate of $22.2 \%$ compared with 7.5\%). A significant main effect of target type was also observed $\left(\mathrm{F}_{1,18}=\right.$ $56.94, \mathrm{p}<0.001$ ), with error rates for expanding targets being significantly lower than those for static targets (a 52.4\% reduction in errors with expansion, from an overall mean of $20.2 \%$ to $9.6 \%$ ). The interaction between target type and age was not significant $\left(\mathrm{F}_{1,18}=4.04, \mathrm{p}=0.06\right)$. Separate analyses of errors showed a significant effect of target type for both older users $\left(\mathrm{F}_{1,9}=57.12, \mathrm{p}<0.001\right)$ and younger users $\left(\mathrm{F}_{1,9}=12.77\right.$, $\mathrm{p}=0.006$ ); for both age groups, performance was significantly improved with target expansion.

There was a significant main effect of $I D\left(\mathrm{~F}_{10,180}=42.89, \mathrm{p}<0.001\right)$ and a significant interaction between $I D$ and age $\left(\mathrm{F}_{10,180}=7.77, \mathrm{p}<0.001\right)$, with older adults appearing to be more affected by increasing task difficulty than younger users (Figure 3). There was also a significant interaction between $I D$ and target type $\left(\mathrm{F}_{10,180}=9.37, \mathrm{p}<\right.$ 0.001). Figure 3 suggests that participants experienced a greater performance gain with target expansion as the $I D$ increased. 
Table III. Subjective Feedback (A value of 4 indicates a neutral response, and lower values favor expanding targets)

\begin{tabular}{|l|c|c|c|c|c|c|c|}
\hline & Ease & Speed & Accuracy & Vis Appeal & Distracting & Annoying & Pref \\
\hline Older \\
\hline Median & 2 & 2 & 1.5 & 2.5 & 1.5 & 1.5 & 1.5 \\
\hline Min & 1 & 1 & 1 & 1 & 1 & 1 & 1 \\
\hline Max & 3 & 3 & 3 & 5 & 3 & 3 & 4 \\
\hline Younger \\
\hline Median & 1 & 1.5 & 1.5 & 3 & 2 & 2 & 2.5 \\
\hline Min & 1 & 1 & 1 & 2 & 1 & 1 & 1 \\
\hline Max & 2 & 3 & 3 & 4 & 5 & 4 & 4 \\
\hline
\end{tabular}

3.6.3. Subjective Feedback. Participants were also asked for subjective responses to the expansion technique. The responses are summarized in Table III where a value of 4 indicates a neutral response, and lower values favor expanding targets. Users' responses to perceived ease, speed and accuracy when using expanded targets compared to static targets were favorable. Both older and younger users perceived expanding targets to be easier (median (older) $=2.0$, median (younger $)=1$ ); faster (median (older) $=2$, median $($ younger $)=1.5$ ); and more accurate (median $($ older $)=1.5$, median $($ younger $)=$ 1.5) than static targets. This is consistent with the results from the objective measures. In addition to the comparative evaluations, users' feedback about specific aspects of the target expansion was also favorable. Both older and younger users indicated that the expansion was visually appealing (median (older) $=2.5$, median (younger) $=3$ ); not distracting (median (older) $=1.5$, median (younger) $=2$ ); nor annoying (median $($ older $)=1.5$, median (younger) $=2$ ); and both older users and younger users showed a preference for expanding targets compared to static targets (median (older) $=1.5$, median (younger) $=2.5$ )

\subsection{Summary}

For the particular implementation of expanding targets studied here (i.e., where only the target to be selected expanded, expansion was to twice the original width of the target, expansion point was $\mathrm{P}=0.5$, and the expansion period was $100 \mathrm{~ms}$ ) and the particular selection task studied here (i.e., a multidirectional arrangement of circular targets with no spatial overlap of the expanded target with any other target), target expansion improved speed and accuracy for both older and younger adults. Overall, times improved by $13 \%(=200 \mathrm{~ms})$ and error rates were reduced by $52.4 \%$. Participants' subjective feedback about the expansion technique was favorable, and users indicated a preference for the expanding targets over the static ones. Building on these initial results, Study 2 examines the potential of expanding targets further.

\section{STUDY 2}

While the results of Study 1 lend support to the use of target expansion to assist older adults, it investigates only one implementation of target expansion. In this implementation, expansion occurs when the cursor is more than $50 \%$ of the initial distance to the target; in other words, the target is expanded when it is within a radius $r$ of the cursor's position where $r=0.5 \mathrm{D}$. If this technique is applied to an interface with multiple targets, all those within a radius $r$ of the cursor's current position would be expanded, and with a relatively large $r$, this would potentially apply to many of the targets on the screen. This would defeat the purpose of saving screen real estate, and for denser 
arrangements of targets, raises issues of expanding targets occluding or pushing aside adjacent targets.

This second study investigates three methods which could be more applicable for a multiple target interface by reducing the number of targets undergoing expansion at any one time. (1) The first is to expand only those targets that are near the cursor, that is, set $r$ to be small. With sufficient empty space between targets, this would mean that only one target would be expanded at a time. (2) The second is when $r$ is half the original width of the target, such that expansion occurs only when the cursor is inside the target's original unexpanded area. This would ensure that only one target would be expanded at a time, even if there is no empty space between the targets. Importantly, though, the target is expanded only in visual space, and does not give the user a larger motor space (i.e., clickable area). (3) The third method expands a target when the cursor enters the target's original unexpanded area, and the target stays at the expanded size for as long as the cursor stays inside the expanded area. Again, this ensures that only one target is expanded at a time, but in contrast with the previous method, this method provides a larger area to click on. With this last method, screen space can be said to be dynamically allocated to targets; in other words, a pixel on the screen could belong to one expanded target on one occasion, and to an adjacent expanded target on a different occasion, depending on the path the cursor took to reach that pixel. This has also been referred to as widget hysteresis [McGuffin and Balakrishnan 2005], where the cursor starts at a pixel and moves through a closed path back to the starting pixel, yet that pixel would be outside the target at the start of the movement, but inside the target at the end.

Study 2 investigates these three methods of expansion with older and younger adults. The study investigates these techniques using a similar setup to Study 1, where only the target to be selected undergoes expansion. This is in order to gain an understanding of how people fundamentally respond to these techniques, with a view to conducting a follow-up study where undesired targets also expand as the cursor moves around the screen.

\subsection{Participants}

Sixteen older adults (mean age $=71.8$ years, 10 female, all right-handed) and 14 younger adults (mean age $=21.6$ years, 2 female, 1 left-handed) participated in this study. Three of the older adults and 5 of the younger adults had also participated in Study 1. The majority of older participants either used the computer daily, or at least once a week. All of the younger participants used a computer on a daily basis. All participants reported normal or corrected vision, and none had any motor difficulties that restricted their use of computers.

\subsection{Apparatus}

As in Study 1, the study was conducted on a Toshiba laptop (Satellite Pro A120) with a 15.4 inch widescreen, 1280 x 800 TFT display, running Windows XP Pro. An optical computer mouse was used to perform selection tasks.

\subsection{Tasks}

Participants performed serial, multidirectional selection tasks for each condition, using the ISO configuration as in Study 1, except that 17 targets were used instead of 25. The number of targets was reduced to 17 in this study to help keep the length of the experiment within a reasonable timeframe for participants, and to minimize the possibility of fatigue as four conditions were being studied instead of two. 


\subsection{Target Types}

Participants completed selections for four target types - static, visual, expanding, and dynamic - where the latter three are the expansion schemes described earlier.

(1) Static. The targets do not expand.

(2) Visual. The targets are expanded in visual space only. The target expands to twice its original width when the cursor enters the original target area, and shrinks back to its original size once the cursor leaves the original target area. Although the target is bigger visually, the area available for selecting the target is no bigger.

(3) Expanding. The targets are expanded in both visual and motor space. The target expands to twice its original width as in Study 1, but this time the expansion point is much closer to the target, that is, when the cursor is in the area that would be occupied by the expanded target.

(4) Dynamic (referring to the dynamic mapping of screen space to expanded targets). The target expands to twice its original width when the cursor enters the original target area. In contrast with the visual condition, the target then remains at its bigger size for as long as the cursor stays inside the expanded area, thereby giving the user a larger area to click on.

Targets expanded over a 50ms period. This expansion period was reduced from the $100 \mathrm{~ms}$ used in Study 1, because compared to the earlier study, the target does not start to expand until much later in the movement.

\subsection{Design}

The study had a $2 \times 4 \times 8$ design, with age (younger, older) as a between-subjects factor, target type (static, visual, expanding, dynamic) and $I D$ as within-subjects factors.

Selections were carried out in sequences of 17 trials, where the target type and the ID were held constant for the sequence. The number of trials for each study participant was as follows.

4 target types (as described above) $\times$

8 sequences (one at each of $8 \mathrm{D} / \mathrm{W}$ combination) $\times$

17 trials per sequence

$=544$ trials per participant

The $8 \mathrm{D} / \mathrm{W}$ combinations were, in pixels, 300/6, 300/12, 300/24, 300/48, 600/6, 600/12, $600 / 24$, and 660/48, giving IDs ranging from 3.75 to 6.66 bits. Each target type was completed in a block of $8 \mathrm{D} / \mathrm{W}$ sequences presented in random order. The order of presentation of target types was balanced across participants (as far as possible given the 14 younger participants) with a $4 \times 4$ balanced Latin Square. At the start of each block, participants were given a demonstration of the target type and they then completed two practice sequences. Data from the practice sequences were not included in the analysis.

Participants were asked to rank the target types in order of preference. The ranking was performed successively, with participants comparing the first two target types after the second block had been completed, and then updating their rankings after each of the third and fourth blocks.

\subsection{Results}

As in Study 1, movement times (i.e., the time elapsed from the start of the trial to the mouse click) and error rates (i.e., percentage of trials where the user clicked outside 
Table IV. Mean Movement Times in Seconds (with standard errors shown in parentheses)

\begin{tabular}{|l|c|c|c|}
\hline & Older & Younger & Overall \\
\hline Expanding & $1.72 \mathrm{~s}(0.107)$ & $0.88 \mathrm{~s}(0.115)$ & $1.30 \mathrm{~s}(0.079)$ \\
\hline Dynamic & $1.86 \mathrm{~s}(0.100)$ & $0.96 \mathrm{~s}(0.106)$ & $1.41 \mathrm{~s}(0.073)$ \\
\hline Visual & $1.96 \mathrm{~s}(0.109)$ & $1.02 \mathrm{~s}(0.116)$ & $1.49 \mathrm{~s}(0.080)$ \\
\hline Static & $1.99 \mathrm{~s}(0.114)$ & $1.04 \mathrm{~s}(0.122)$ & $1.52 \mathrm{~s}(0.083)$ \\
\hline Overall & $1.9 \mathrm{~s}(0.104)$ & $0.97 \mathrm{~s}(0.111)$ & \\
\hline
\end{tabular}

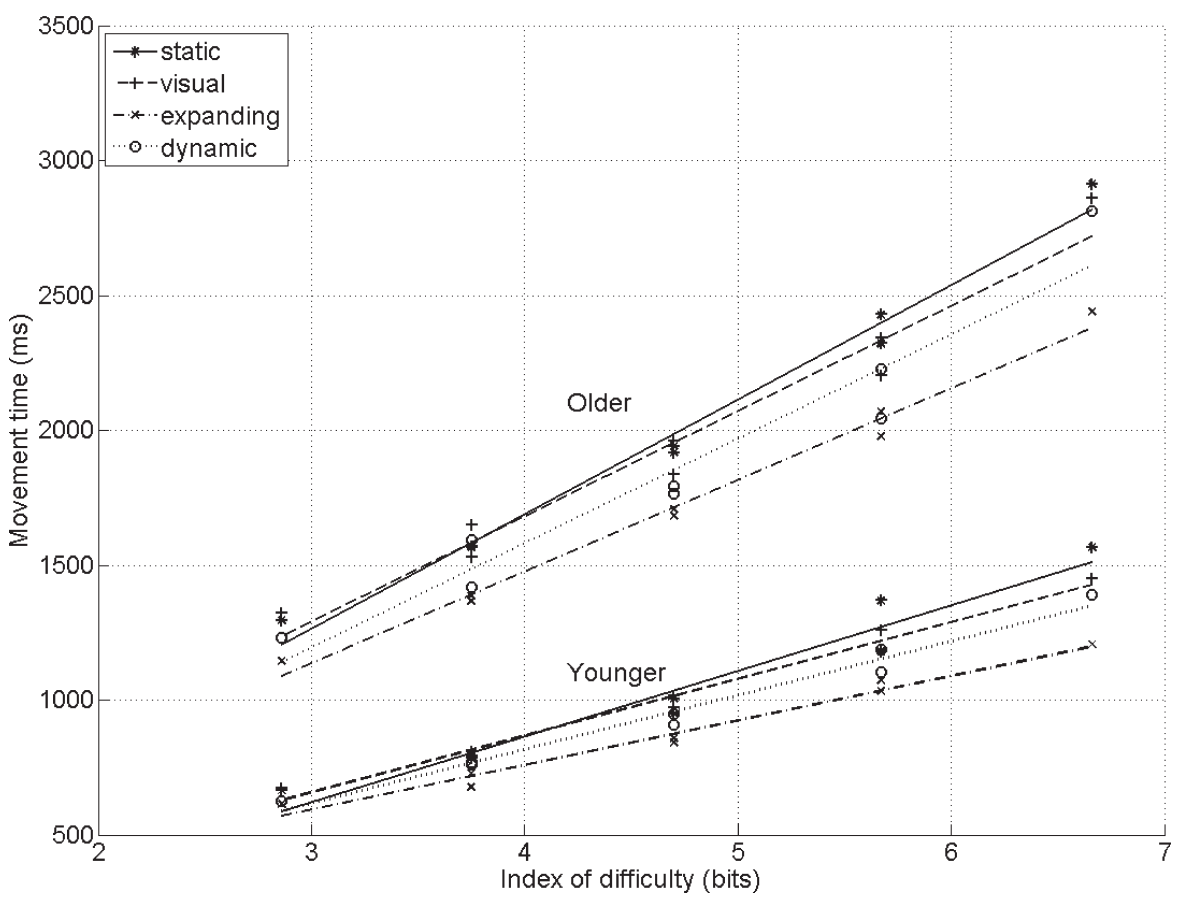

Fig. 4. Movement times versus ID for each target condition, for older and younger participants.

the target) were analyzed with a mixed design ANOVA, with target type and ID as within-subject variables and age as a between-subject variable. The $I D$ for an expanding target was calculated using the initial, unexpanded width.

Trials with movement times greater than three standard deviations from the mean for each condition and for each user were treated as outliers and removed, as were trials that were completed in less than $300 \mathrm{~ms}$. In total, the number of data points removed was less than $1 \%$ of the total number of data points. Due to technical issues, data was missing for one out of the 32 target type $\times I D$ combinations for one younger user and two older users (total of fifty one data points). For each user, the mean of the remaining trials for each condition was then calculated and used in the analysis and the accompanying figures and tables.

4.6.1. Movement Times. Mean movement times are summarized in Table IV and illustrated in Figure 4. The ANOVA showed a significant main effect of age $\left(F_{1,28}=\right.$ $35.95, \mathrm{p}<0.001$ ), with the older users having longer movement times than the younger users $(1.9 \mathrm{~s}$ compared with $0.97 \mathrm{~s})$. A significant main effect of target type was also observed $\left(F_{1.87,52.25}=16.51, \mathrm{p}<0.001\right.$, Greenhouse-Geisser correction), with 
Table V. Mean Error Rates (with standard errors shown in parentheses)

\begin{tabular}{|l|c|c|c|}
\hline & Older & Younger & Overall \\
\hline Expanding & $11.63 \%(3.56 \%)$ & $3.10 \%(1.01 \%)$ & $7.36 \%(1.96 \%)$ \\
\hline Dynamic & $16.41 \%(3.91 \%)$ & $7.25 \%(2.09 \%)$ & $11.83 \%(2.31 \%)$ \\
\hline Visual & $22.01 \%(3.85 \%)$ & $9.14 \%(2.11 \%)$ & $15.58 \%(2.29 \%)$ \\
\hline Static & $21.40 \%(4.18 \%)$ & $8.66 \%(1.94 \%)$ & $15.02 \%(2.41 \%)$ \\
\hline Overall & $17.9 \%(3.63 \%)$ & $7.0 \%(1.71 \%)$ & \\
\hline
\end{tabular}

movement times for the expanding target condition being significantly lower than the other three conditions, and those for the dynamic condition being significantly lower than the static condition. Overall mean movement times were 1.30s for the expanding condition; $1.41 \mathrm{~s}$ for the dynamic condition; $1.49 \mathrm{~s}$ for the visual condition; and $1.52 \mathrm{~s}$ for the static condition. Comparing each of the expanding target types with the static condition, expanding targets gave a $14 \%$ improvement comparable with the improvement seen in Study 1. Dynamic targets provided a smaller improvement in acquisition time, with a 7\% improvement over the static condition, whereas the visual condition provided no significant improvement over the static condition. There was no significant interaction between target type and age $\left(F_{1.87,52.25}=1.123, \mathrm{p}=0.33\right.$, GreenhouseGeisser correction).

Separate analyses of older and younger users again showed a significant main effect of target type (older: $\mathrm{F}_{3,45}=8.201 \mathrm{p}<0.01$; younger: $\mathrm{F}_{3,39}=24.04, \mathrm{p}<0.01$ ). For each age group, the significant pairwise comparisons were the same as when both age groups were analyzed together (all $\mathrm{p}<0.01$ ). Comparing each of the expanding target types with the static condition, for older adults, expanding gave a $14 \%$ improvement $(\mathrm{t}(15)=3.33, \mathrm{p}=0.005)$; dynamic gave $7 \%(\mathrm{t}(15)=2.35, \mathrm{p}=0.033)$; and visual gave no improvement. The results were similar for the younger users with expanding targets giving a $15 \%$ improvement $(\mathrm{t}(13)=5.88, \mathrm{p}<0.01) ;$ dynamic targets giving an $8 \%$ improvement $(\mathrm{t}(13)=3.56, \mathrm{p}=0.04)$; and visual providing no improvement.

There was a significant main effect of $I D\left(F_{1.92,53.76}=129.00, \mathrm{p}<0.001\right.$, Greenhouse-Geisser correction), and a significant interaction between $I D$ and age $\left(F_{1.92,53.76}=12.46, \mathrm{p}<0.001\right.$, Greenhouse-Geisser correction), with movement times for older users appearing to be more affected by increasing task difficulty than for younger users. There was also a significant interaction between ID and target type $\left(F_{5.74,160.76}=2.58, \mathrm{p}=0.022\right.$, Greenhouse-Geisser correction). Figure 4 suggests that part of this interaction may be that expanding targets are less affected by increasing ID than static targets, as indicated by the differences in the slopes of the lines.

4.6.2. Error Rates. An error was defined to occur when the user clicked outside the target. For each user, error rates for each condition (i.e., the percentage of trials in each condition that resulted in an error) were calculated and used in the analysis. Table $\mathrm{V}$ and Figures 5 and 6 show the mean error rates across all participants, for older and younger users, respectively, and for each of the conditions.

There was a significant main effect of age $\left(F_{1,28}=6.63, \mathrm{p}=0.016\right)$, with the older users making more than twice as many errors overall than the younger ones. Overall mean error rates were $17.9 \%$ for the older users and $7.0 \%$ for the younger ones. A significant main effect of target type was also observed $\left(F_{2.44,68.3}=16.47, \mathrm{p}<0.001\right.$, Greenhouse-Geisser correction), with error rates for the expanding and dynamic conditions being significantly lower than for the static condition. Overall mean error rates were reduced from $15.02 \%$ for the static condition to $7.36 \%$ for the expanding condition (an improvement of 50.9\%, comparable with Study 1), and to $11.83 \%$ for the dynamic condition (an improvement of $21.24 \%$ ). The interaction between target 


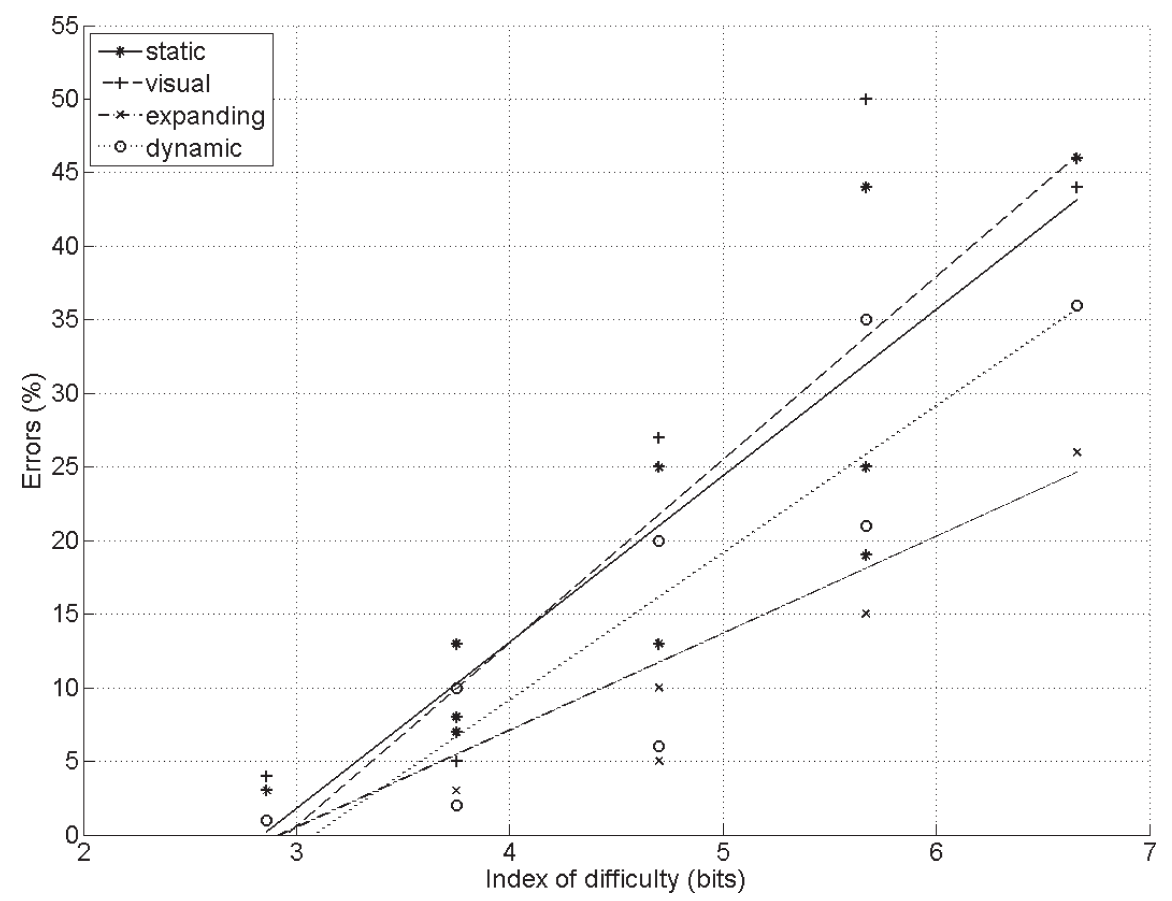

Fig. 5. Mean error rates versus ID for older participants for each of the conditions.

type and age was insignificant $\left(F_{2.44,68.3}=1.52, \mathrm{p}=0.222\right.$, Greenhouse-Geisser correction).

Separate analyses of older and younger users again showed a significant main effect of target type (older: $\mathrm{F}_{3,45}=9.328 \mathrm{p}<0.01$; younger: $\mathrm{F}_{3,39}=24.41, \mathrm{p}<0.01$ ). For each age group, the significant pairwise comparisons were the same as when both age groups were analyzed together (all $\mathrm{p}<0.01$ ). Comparing each of the expanding target types with the static condition, for older adults; expanding gave a $46 \%$ improvement $(\mathrm{t}(15)=3.33, \mathrm{p}=0.005) ;$ dynamic gave a $23 \%$ improvement $(\mathrm{t}(15)=2.20, \mathrm{p}=0.04)$; and visual gave no improvement. The results for the younger users only showed a significant improvement for expanding targets, with expanding targets giving a $64 \%$ improvement $(\mathrm{t}(13)=4.59, \mathrm{p}=0.01)$; dynamic and visual targets gave no improvement over static targets.

There was a significant main effect of $I D\left(F_{1.55,43.35}=35.76, \mathrm{p}<0.001\right.$, GreenhouseGeisser correction), with an overall trend of error rates increasing with task difficulty. However, it should be noted that although the trend was an increase in error rate as the $I D$ increased, where two different $\mathrm{D} / \mathrm{W}$ combinations were used at the same $I D$, the number of errors was higher for the $\mathrm{D} / \mathrm{W}$ combination with the smaller target width. As an example, for an ID of 5.67 with the expanding condition for the older participants, the combination $300 / 6$ produced a higher error rate (mean $=25 \%$ ) than $600 / 12$ (mean $=15 \%$ ), suggesting that target width (at least for smaller targets) has a greater effect on accuracy than the distance to the target.

There was a significant interaction between $I D$ and age $\left(F_{1.55,43.35}=10.24, \mathrm{p}=\right.$ 0.001, Greenhouse-Geisser correction), with error rates for older users appearing to increase more with an increasing level of task difficulty than for younger users. There was also a significant interaction between $I D$ and target type $\left(F_{8.49,237.65}=\right.$ $4.12, \mathrm{p}<0.001$, Greenhouse-Geisser correction). As with movement times, part of this 


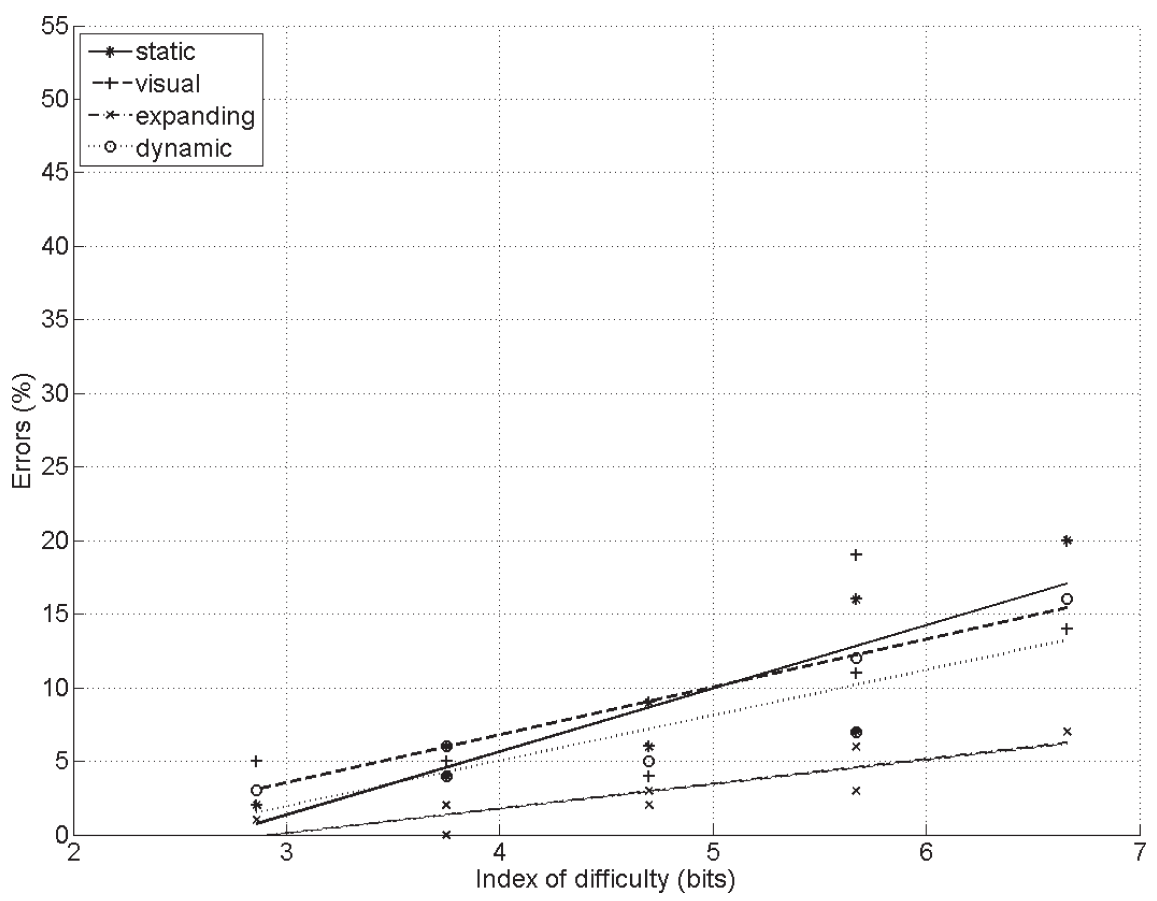

Fig. 6. Mean error rates versus ID for younger participants for each of the conditions.

interaction may be that expanding targets are less affected by increasing $I D$ than static targets.

4.6.3. Subjective Feedback. Regarding participants' subjective rankings of the different target types, Figure 7 shows an agreement amongst younger participants in the preferred target type. Twelve of the 14 younger participants preferred the expanding target type; one participant opted for the static target and one did not provide any preference for a specific target type, and so their response is not included in the figure. Seven of the 16 older users also preferred the expanding target condition, but compared with the younger users, the older adults showed a greater spread over the other three conditions. Two older participants ranked all target types the same, and so their responses are not indicated in the figure.

\subsection{Summary of Results}

This study compared static targets with three alternative techniques for expansion. Expanding targets enlarged the target in both visual and motor space before the cursor reached the original target, and improved performance for both older and younger groups; compared with the static condition, overall, movement times were reduced by $0.22 \mathrm{~s}$ ( $14 \%$ improvement) and error rates by $7.8 \%$ (50.1\% improvement). Expanding targets were also ranked as the most preferred target type by 7 out of 16 older participants and 12 out of 14 younger participants.

Dynamic targets enlarged the target in both visual and motor space after the cursor reached the original target, and provided an improvement over static targets for both age groups; overall, times were reduced by $0.11 \mathrm{~s}$ (7\% improvement) and error rates by $3.3 \%$ (21.4\% improvement). 


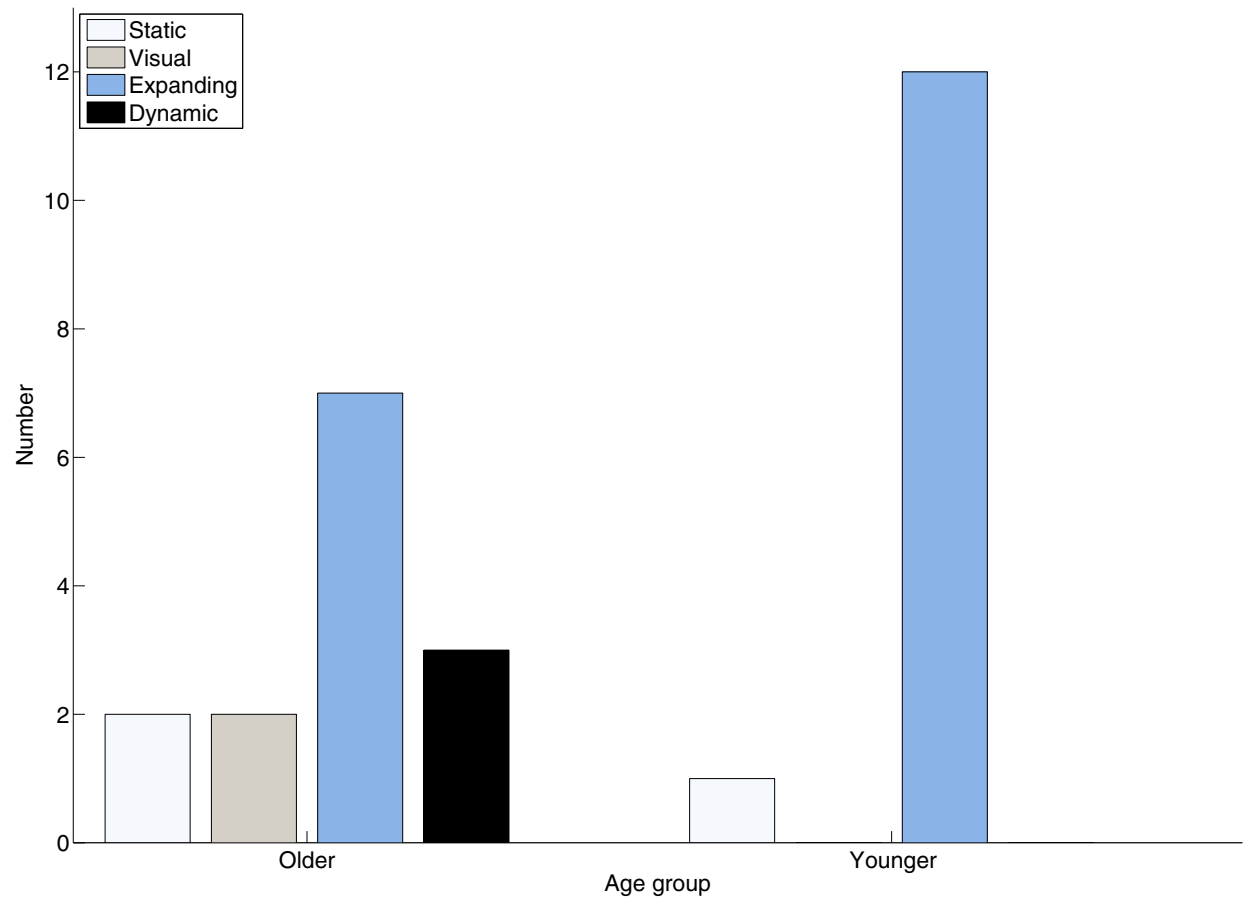

Fig. 7. Target types ranked as first choice by older and younger participants.

Visual targets enlarged the target in visual space only, and was found to provide no performance benefits over static targets.

Finally, while movement times and error rates generally increased with increasing task difficulty for all target types, results suggest that expanding targets may be less affected by increasing ID than static targets, and as a result the more difficult the task, the greater the benefit gained from the expansion.

\subsection{Discussion}

This article has presented two studies, both of which were concerned with improving target selection for older adults through the technique of expanding targets. Study 1 compared two types of targets: static targets and expanding targets, where a target expanded when the cursor crossed over $50 \%$ of the initial distance to the target. Study 2 investigated target expansion further and compared static targets with three different implementations of expanding targets.

4.8.1. Performance. Study 1 and Study 2 both showed performance differences between older and younger users. In both studies, older users were found to take about twice as long for target selection and to have error rates that were 2.55 and 2.96 times higher than the younger group. In addition, both studies found a significant interaction between age and index of difficulty, and the data suggest that older adults experience a greater decline in performance with increasing task difficulty (i.e., increasing ID) than younger users. These results are consistent with previous studies on age-related differences in general target selection (e.g., Worden et al. [1997], Ketcham et al. [2002, 2004]), in which the differences were attributed to declines in visual abilities and motor skills, and a consequent adjustment in the submovement structure of cursor movement [Walker et al. 1997]. With respect to previous studies 
on age-related differences in selection of expanding targets, both Bohan and Scarlett [2003] and Lee et al. [2012] also reported that older adults took significantly longer than younger adults, although Lee et al. [2012] found no overall significant difference in terms of errors (errors were not reported in Bohan and Scarlett [2003]).

The results of both our studies show that target expansion can improve time and accuracy for older adults. Study 1 found that overall times were reduced by $200 \mathrm{~ms}$ ( $13 \%$ improvement) and errors were reduced by $14.7 \%$ (52.4\% improvement). In Study 2, for expanding and dynamic targets, respectively, overall times were reduced by $216 \mathrm{~ms}$ and $105 \mathrm{~ms}$ (14.2\% and $7 \%$ improvement), and errors by $7.8 \%$ and $3.3 \%(50.5 \%$ and $21.4 \%$ improvement). These time improvements are comparable to the gains reported in McGuffin and Balakrishnan [2005], Zhai et al. [2003], Cockburn and Brock [2006], which were between 6-12\%. However, our findings contrast with those of Lee et al. [2012] who did not find any significant performance improvement for older adults when expansion was used. This is discussed further next.

The literature on target expansion makes a distinction between untiled and tiled arrangements of targets. Untiled arrangements have space between the targets (e.g., sparsely spaced icons on a virtual desktop); tiled arrangements have little or no space between the targets (e.g., toolbars, ribbons, and spreadsheets [Ruiz and Lank 2010]). The studies reported in this article examine the former. In contrast, Lee et al. [2012] focus on the latter, where their experimental stimulus comprised groups of $6,9,18$, 27 , and 36 icons arranged in 3 rows. The groups were arranged side by side to form a palette of $3 \times 38=114$ icons, with a 5-pixel space between groups, 0 pixel space between icons, and each icon was 18 x 18 pixels in size. The challenges with implementing target expansion in tiled arrangements, particularly in addressing the occlusion and displacement of neighboring targets, have been discussed in the literature [McGuffin and Balakrishnan 2005; Ruiz and Lank 2010], and it has been noted that successful implementation of expansion for tiled arrangements remains an "open challenge" [Ruiz and Lank 2010]. Hence, the contrast between our results and those of Lee et al. [2012] may be less about differences in whether older adults can benefit from target expansion, and more about differences in implementing target expansion for untiled versus tiled targets, which is an area being investigated more generally. The investigation of untiled targets has merit in its own right, and indeed the majority of selection facilitation techniques have only been investigated for untiled targets [McGuffin and Balakrishnan 2005], and so the results from the current two studies add to this body of work and provide evidence of the benefits of target expansion for older adults for untiled targets.

The effects of target expansion on error rates vary in the literature, and are difficult to compare directly due to differences in the task (i.e., whether or not participants were allowed multiple clicks in a trial in order to select a target), and hence how errors and error rates were defined and calculated. Bearing these differences in mind, previously reported results had no significant effect [Cockburn and Brock 2006; Lee et al. 2012], reductions in error rates of approximately $2.2 \%$ (73\% improvement) [Zhai et al. 2003] and $1.2 \%$ (42\% improvement) [McGuffin and Balakrishnan 2005] for lower task difficulties, and increases in error rates of $2.8 \%$ (39\% decrease in performance) [Zhai et al. 2003] and 3.2\% (88\% decrease in performance) [McGuffin and Balakrishnan 2005] for higher task difficulties. Our results provide further support for the idea that expanding targets can improve error rates, but in light of the range of results reported in the literature, this is an area that warrants further study.

Where target expansion has been reported to improve error rates, the relative improvement is greater for errors than for times. In our studies, error rates were improved by about $50 \%$, compared with times that improved by about $14 \%$. Hence there may be greater merit in using target expansion as a method of reducing errors more so 
than of improving selection times, particularly as older adults tend to be more erroraverse than younger adults, and have more difficulties recovering from errors [Mead et al. 1999].

As noted by Lee et al. [2012], designs for target expansion vary, and it is useful to investigate performance across different implementations. Our results show that expansion can improve times and errors for older adults for three different implementations of the expansion, but also that the degree of improvement varies across them. With the relatively conservative implementation of Study 1, where the target expanded to twice its width when the cursor was halfway to the target, times and errors improved by $13 \%$ and $52.4 \%$, respectively. With the expanding implementation in Study 2, where the target still expanded to twice the original width but where the expansion occurred much later in the movement, that is, not until the cursor was within the expanded area, there was a $14 \%$ improvement in times and a 50\% improvement in errors. Thus, despite the later expansion point, older adults gained the same level of benefit as when the expansion occurred much earlier in the movement. This contrasts with Bohan and Scarlett's [2003] result that their participants saw no benefits from late expansion, but their implementation of the expansion was quite different to ours. In theirs, once the cursor was inside the expansion area, the size of the target was inversely proportional to the distance of the cursor from the target's center. So at cursor positions just inside the expansion border, the target would be more or less at its original size, and the target would only approach its fully-expanded size when the cursor was near the target's center. In contrast, with our implementation, once the cursor had crossed the expansion point, the user had the fully-expanded area available to them, and were able to benefit from this. This is useful, since late expansion is proposed as more practical and useful for a real interface [McGuffin and Balakrishnan 2005]. With the dynamic implementation in Study 2, where the expansion did not occur until after the cursor was inside the original target space, there was a $7 \%$ improvement in times and $21.4 \%$ improvement in errors. It is particularly interesting that participants still benefited from this method, even though their cursor was already inside the original, smaller target area. Again there was a greater benefit for error reduction than there was for time improvement. The performance gains might be the result of having a larger motor space in which to perform corrective submovements, and a reduction in slipping-off errors, both of which have been observed, particularly in older adults [Paradise et al. 2005].

Although others have suggested that the visual feedback alone can have beneficial effects [Cockburn and Brock 2006], in our study, visual targets provided no performance benefits. It may be that with older adults, as compared with younger participants, a larger proportion of the benefit is due to the enlarged motor space rather than just the visual cue of the expansion, and this may be of help with age-related declines in motor control.

Although the techniques in Study 2 were designed to be implementable in multiple target scenarios, we have only investigated them here in single-target tasks. Empirical studies of these techniques with more realistic interfaces would be an important area of future work, to see how well they would apply in the real world, see Section 4.8.4.

4.8.2. Spatial Distribution of Mouse Clicks. The spatial distribution of mouse clicks was examined, to see how participants were making use of the expanded target area. Figure 8 shows the spatial distribution of mouse clicks that occurred within five times the target's radius in Study 1. The distance to the target's center has been normalized by dividing by one half of the initial width of the respective target, so that the x-axis is in units of the target's radius (W/2). For static targets, clicks between 0 and 1 radius 


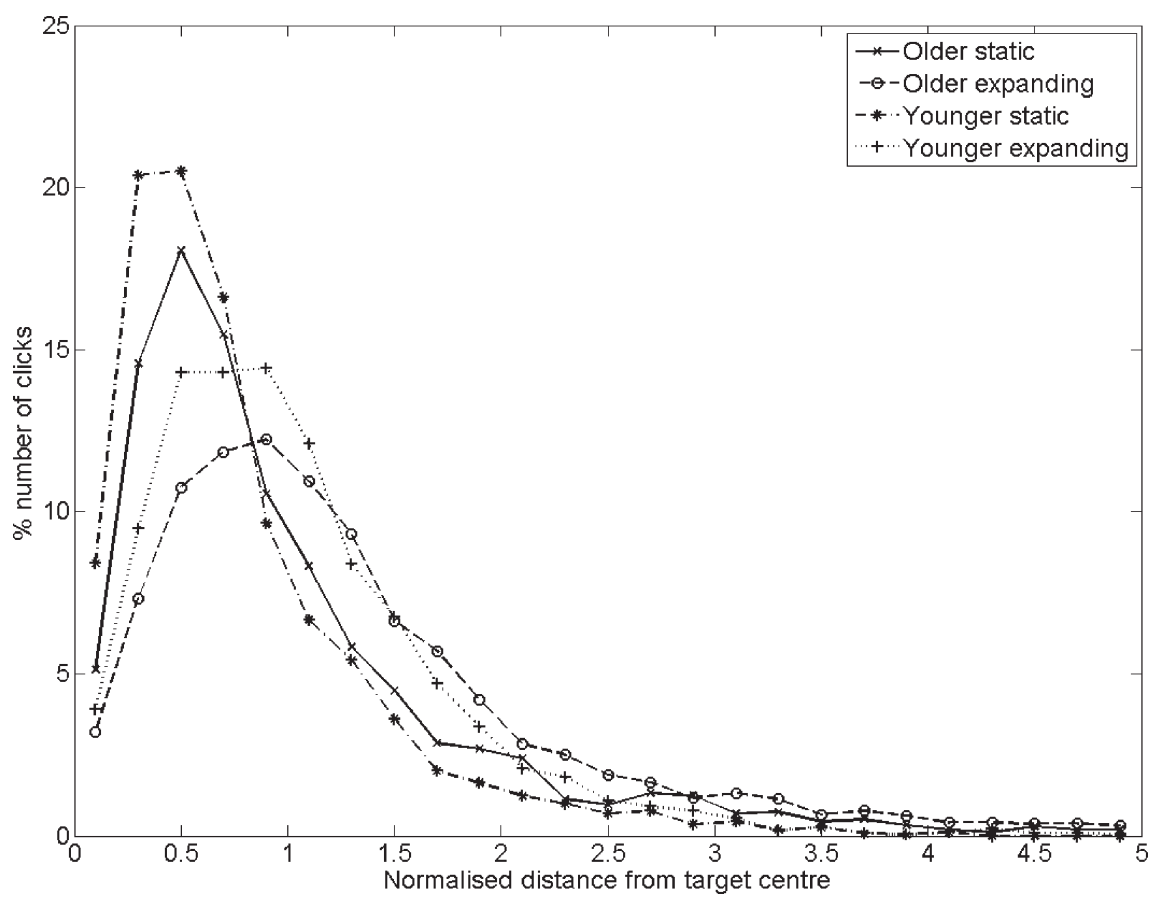

Fig. 8. Spatial distribution of mouse clicks that occurred within five times the target's radius for Study 1.

from the target's center "hit" the target, and the figure illustrates a concentration of clicks inside the selectable target area. The figure also illustrates that a high proportion of the errors occur mainly just outside the target and tail-off with distance from the target's center, and that compared with younger adults, the older adults have a slightly higher proportion of errors between 1 to 2 radii from the target's center. For expanding targets, the concentration of clicks inside the initial target area flattens out, and the clicks spread into the expanded target area, that is, between 1 and 2 radii from the target's center. This is observed for both older and younger users, and illustrates that both groups were making use of the enlarged target width. Zhai et al. [2003] reported similar findings with younger users.

In Study 2 (Figures 9 and 10), we see again a concentration of clicks inside the unexpanded area (i.e., within one radius from the center of the target). Particularly for the expanding target condition, but also to a certain degree for the dynamic target condition, a larger proportion of mouse clicks take place in the expanded area (i.e., between 1 to 2 radii from the target center) as compared with the other two conditions. This again illustrates that participants were making use of the expanded target area, and hence benefiting from the additional motor space and not just the visual cues alone, as Cockburn and Brock [2006] suggested. The effect is more pronounced for the expanding condition than the dynamic one, so having the expansion happen before the cursor reaches the original target area enables users to take better advantage of the enlarged space, but it is interesting that, in the dynamic condition this is still observed albeit to a lesser extent, even though their cursor had already entered the original target region.

4.8.3. Subjective Feedback from Participants. Alongside performance measures, users' subjective responses are also an important factor in investigating the appropriateness 


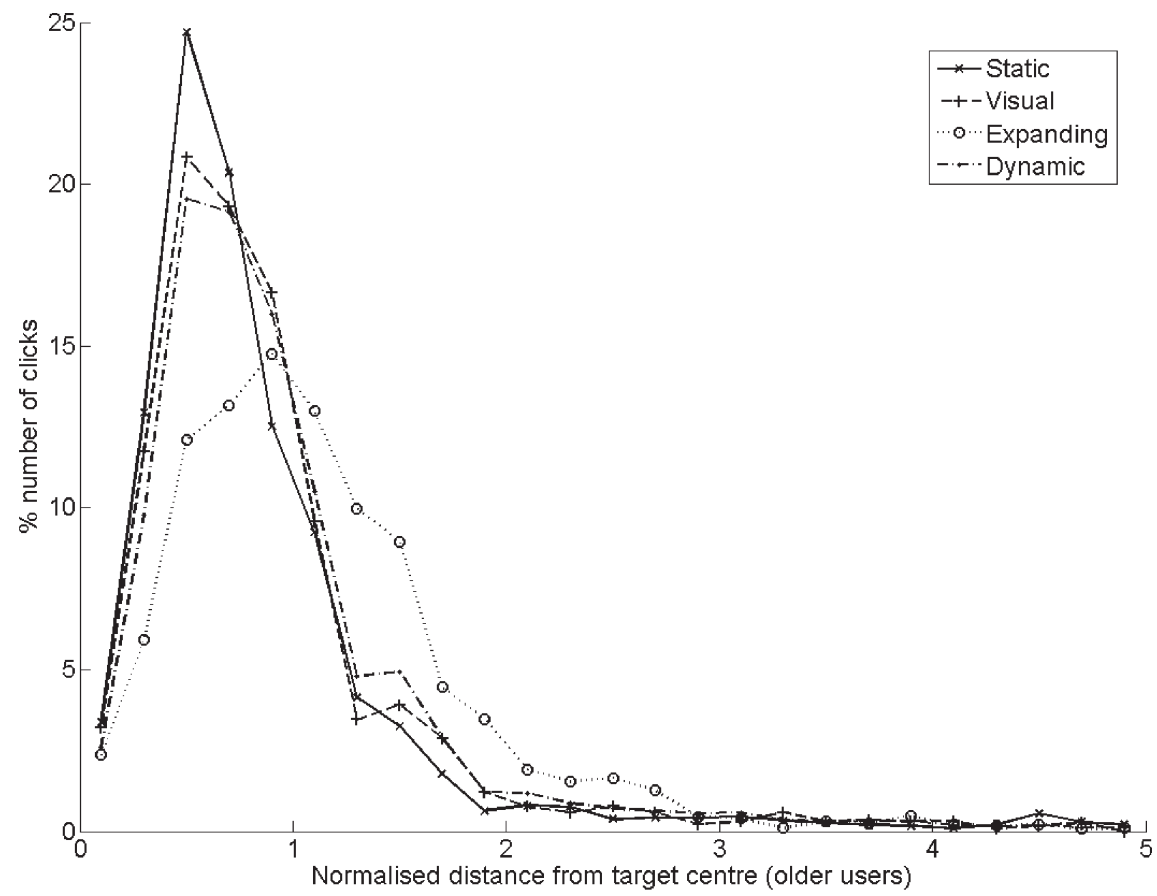

Fig. 9. Spatial distribution of mouse clicks (as a percentage of total number of clicks over all users) for older users that occurred within five times the target's radius.

of the use of target expansion in an interface. In both of these studies, participants' responses indicate that the improvements in performance with expanding targets were not at the cost of user satisfaction, and hence lends further support for the use of expanding targets in user interfaces. In Study 1, both older and younger participants expressed a favorable opinion of expanding targets, and perceived them to be easier and faster to select and less prone to errors, and neither found the expansion distracting or annoying. In Study 2, expanding targets again ranked highly. Comments from some of the older adults provide some indication of what they liked about the expansion.

"I found the expansions helpful. I found the statics [targets] needed all my concentration."

"Expanding target easier to see."

"Expansion directs eye towards target."

Although responses about expansion were generally favorable, one older participant was more ambivalent:

"Did not feel it [expanding targets] made a great deal of difference - for me, size was most important,"

and one younger participant did not like target expansion as a technique in itself, but suggested that it was useful as a method for determining whether the mouse was within the selection area.

"Expansion was offputting ... but for smaller targets the expansion was very useful to see whether or not [the] mouse was on [the] target." 


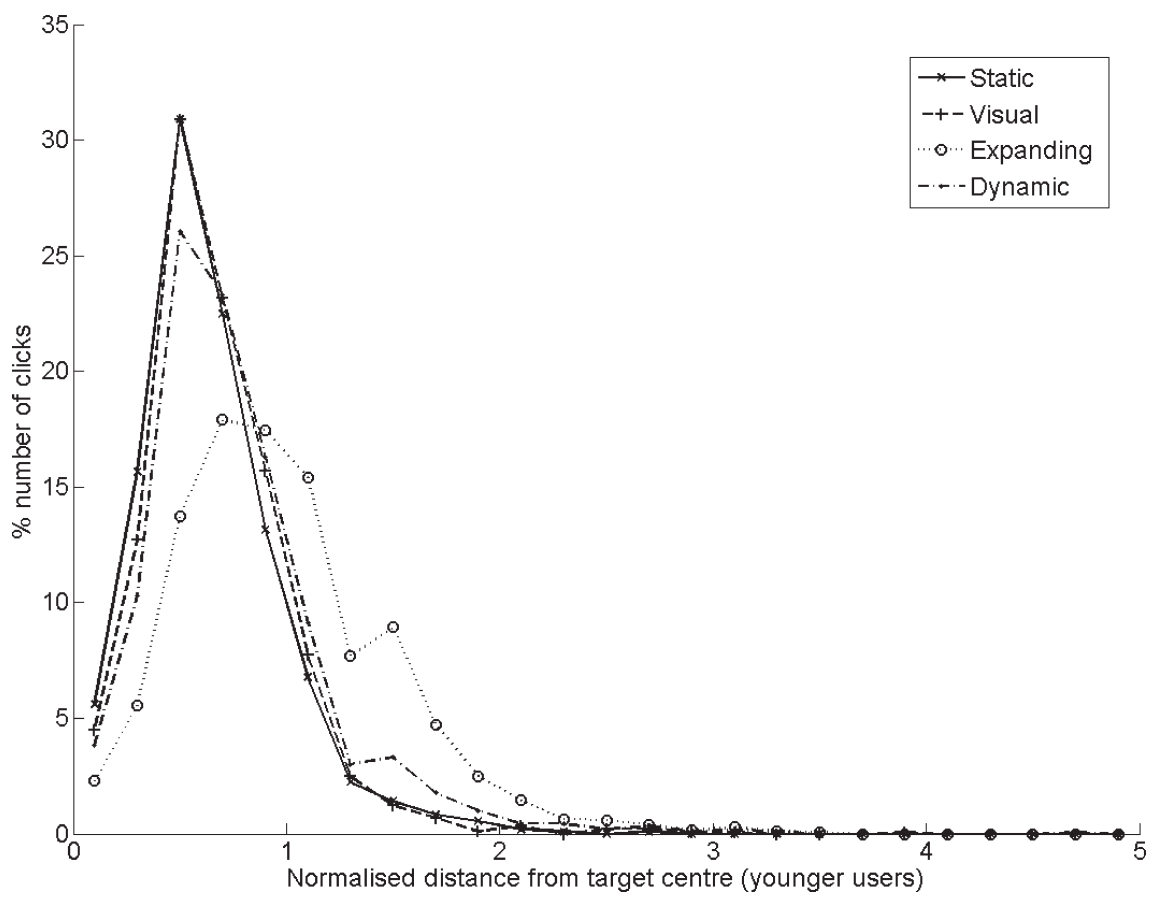

Fig. 10. Spatial distribution of mouse clicks (as a percentage of total number of clicks over all users) for younger users that occurred within five times the target's radius.

The advantages for this participant, then, might equally be achieved with other techniques, such as target highlighting [Cockburn and Brock 2006].

With the younger participants, there were also reservations about how well target expansion would work in a typical Windows interface, as opposed to the experimental task. They felt that the benefits of expansion might be countered by potential limitations such as occlusion of the target or false expansion of targets that were not meant to be selected.

"Expansion much easier to use ... but could get annoying quite quickly"

"If other objects are found on the screen ... then I may be distracted"

In contrast, older users did not express any such concerns about using expanding targets in a Windows interface. However, it is not clear whether this is because they considered the benefits of expansion to outweigh any potential problems, or whether they had not considered the limitations of using expanding targets in a realistic interface. Nevertheless, we recognize that the investigation of these techniques in more realistic interfaces, with both older and younger participants, is an area that requires further investigation.

4.8.4. Real-World Application. These studies provide a useful starting point for understanding the effects of target expansion on selection performance of older versus younger users. With regards to applying this technique to real-world interfaces, however, further work is required. The targets in this study were circular and arranged in a very controlled layout based on the ISO 9241-9 standard. In realistic interfaces, targets take a variety of shapes, sizes, and layouts, which raise additional questions about 
how expansion should be implemented. For example, implementing target expansion for tiled arrangements remains an open challenge (as discussed in Section 4.8.1). Even for untiled arrangements, the implementations studied here presume knowledge about which is the desired target (i.e., only the target to be selected expanded, all others remained static in size); yet this is very difficult to determine in practice. One possibility is to use the methods from Study 2 to expand each target that the cursor approaches, whether the target is desired or not. The effect of this potential distraction on users' performance and satisfaction requires investigation. Other studies have tried to predict which target the user is aiming at, using methods such as kinematic endpoint prediction [Ruiz and Lank 2010]. Creating accurate prediction models still remains a challenge, nor have they been developed and investigated with older adults, and they additionally raise the question that if an accurate prediction is possible, then why not select the target for the user without any further need for expansion or other facilitation techniques.

In addition to knowing the user's desired target, target expansion requires more general knowledge of where targets are on the screen, that is, it is a target-aware technique. Some target-agnostic techniques have aimed to get around this issue via mouse movement and click behavior (without requiring knowledge of targets) including PointAssist [Hourcade et al. 2008, 2010], the Angle Mouse [Wobbrock et al. 2009], and the Pointing Magnifier [Jansen et al. 2011]. However, target-aware techniques outperform target-agnostic ones [Dixon et al. 2012], and so there are still efforts being made to implement target-aware techniques in real-world interfaces. One important challenge is that often external software does not have access to the size and location of interface elements on the screen [Dixon et al. 2012]. To implement target expansion in a real-world context, this issue would need to be addressed; for example, one possible technique is pixel-level analysis of the graphical user interface [Dixon et al. 2012].

\section{CONCLUSIONS}

Expanding targets have good potential as a method of improving target selection performance for older adults. Results from our two studies show that for pointing and clicking tasks with a computer mouse under experimental conditions, expansion can provide improvements in time by up to $14 \%$, and improvement in error rate reduction of up to $50 \%$. Additionally, expanding targets are beneficial even when the expansion happens late in the movement, that is, after the cursor has reached the expanded target area, or even after it has reached the original target area. Although the magnitude of the effect is smaller in the latter case, it has the advantage of being more applicable in interfaces where there is no space between adjacent targets. Participants' subjective feedback on target expansion was generally favorable for these experimental tasks, and this lends further support for using the technique. However, younger participants also expressed some reservations about the potential for the expansion to be distracting and annoying in a real interface. Further work with older adults is required to investigate which expansion implementations are practicable in a real interface, and their effects on performance and user satisfaction. Additionally, technical challenges with implementing target-aware techniques in real-world interfaces would need to be addressed.

\section{ACKNOWLEDGMENTS}

We thank our study participants, and the staff and volunteers of AgeUK, Berkshire and AgeUK, Reading. We also thank the reviewers for their helpful suggestions. 


\section{REFERENCES}

Ahlström, D., Hitz, M., and Leitner, G. 2006. An evaluation of sticky and force enhanced targets in multi target situations. In Proceedings of the 4th Nordic Conference on Human-Computer Interaction: Changing Roles (NordiCHI'06). ACM, New York, 58-67.

Blanch, R. and Ortega, M. 2009. Rake cursor: Improving pointing performance with concurrent input channels. In Proceedings of the SIGCHI Conference on Human Factors in Computing Systems (CHI'09). ACM, New York,1415-1418.

Blanch, R., Guiard, Y., and Beaudouin-Lafon, M. 2004. Semantic pointing: Improving target acquisition with control-display ratio adaptation. In Proceedings of the SIGCHI Conference on Human Factors in Computing Systems (CHI'04). ACM, New York, 519-526.

Bohan, M. and Scarlett, D. 2003. Can expanding targets make object selection easier for older adults? Usability News 5.1. http://usabilitynews.org/can-expanding-targets-make-object-selection-easier-for-older-adults.

Chaparro, A., Bohan, M., Fernandez, J., Choi, S., and Kattel, B. 1999. The impact of age on computer input device use: Psychophysical and physiological measures. Int. J. Ind. Ergonomics 24, 5, 503-513.

Cockburn, A. and Brewster, S. A. 2005. Multimodal feedback for the acquisition of small targets. Ergonomics $48,9,1129-1150$.

Cockburn, A. and Brock, P. 2006. Human on-line response to visual and motor target expansion. In Proceedings of Graphics Interface'06, 81-87.

Czaja, S. J. and Lee, C. C. 2003. Designing computer system for older adults. In Handbook of HumanComputer Interaction, J. Jacko and A. Sears Eds., Lawrence Erlbaum and Associates, New York, 413-428.

Dickinson, A., Eisma, R., and Gregor, P. 2011. The barriers that older novices encounter to computer use. Universal Access Inf. Soc. 10, 3, 261-266.

Dixon, M., Fogarty, J., and Wobbrock, J. 2012. A general-purpose target-aware pointing enhancement using pixel-level analysis of graphical interfaces. In Proceedings of the 2012 ACM Annual Conference on Human Factors in Computing Systems (CHI'12). ACM, New York, 3167-3176.

Grossman, T. and Balakrishnan, R. 2005. The bubble cursor: Enhancing target acquisition by dynamic resizing of the cursor's activation area. In Proceedings of the SIGCHI Conference on Human Factors in Computing Systems (CHI'05). ACM, New York, 281-290.

Guiard, Y., Blanch, R., and Beaudouin-Lafon, M. 2004. Object pointing: A complement to bitmap pointing in GUIs. In Proceedings of Graphics Interface (GI'04). 9-16.

Hollinworth, N. and Hwang, F. 2011. Cursor relocation techniques to help older adults find 'lost' cursors. In Proceedings of the SIGCHI Conference on Human Factors in Computing Systems (CHI'11). ACM, New York, 863-866.

Hourcade, J. P. and Berkel, T. R. 2008. Simple pen interaction performance of young and older adults using handheld computers. Interact. Comput. 20, 1, 166-183.

Hourcade, J. P., Perry, K. B., and Sharma, A. 2008. PointAssist: Helping four year olds point with ease. In Proceedings of the 7th International Conference on Interaction Design and Children (IDC'08). ACM, New York, 202-209.

Hourcade, J. P., Nguyen, C. M., Perry, K. B., and Denburg, N. L. 2010. Pointassist for older adults: Analyzing sub-movement characteristics to aid in pointing tasks. In Proceedings of the SIGCHI Conference on Human Factors in Computing Systems (CHI'10). ACM, New York, 1115-1124.

Hwang, F., Batson, H., and Williams, N. 2008. Bringing the target to the cursor: Proxy targets for older adults. In CHI'08 Extended Abstracts on Human Factors in Computing Systems (CHI EA'08). ACM, New York, 2775-2780.

ISO 9241. 2000. Ergonomic requirements for office work with visual display terminals (VDTs)—part 9, requirements for non-keyboard input devices. International Organization for Standardization.

Jansen, A., Findlater, L., and Wobbrock, J. O. 2011. From the lab to the world: Lessons from extending a pointing technique for real-world use. In CHI'11: Extended Abstracts on Human Factors in Computing Systems. ACM, New York, 1867-1872.

Keates, S. and Trewin, S. 2005. Effect of age and Parkinson's disease on cursor positioning using a mouse. In Proceedings of the 7th International ACM SIGACCESS Conference on Computers and Accessibility. ACM, New York, 68-75.

Ketcham, C. J. and Stelmach, G. 2004. Movement control in the older adult. In Technology for Adaptive Aging, R. Pew and S.Van Hemel Eds., National Academies Press, Washington, D.C., 64-92. 
Ketcham, C. J., Seidler, R. D., Van Gemmert, A. W., and Stelmach, G. E. 2002. Age-related kinematic differences as influenced by task difficulty, target size, and movement amplitude. J. Gerontology: Psychol. Sci. 57B, 1, P54-P64.

Kobayashi, M. and Igarashi, T. 2008. Ninja cursors: Using multiple cursors to assist target acquisition on large screens. In Proceedings of the SIGCHI Conference on Human Factors in Computing Systems (CHI'08). ACM, New York, 949-958.

Lee, D., Kwon, S., and Chung, M. K. 2012. Effects of user age and target-expansion methods on targetacquisition tasks using a mouse. Appl. Ergonomics 43, 1, 166-175.

McGuffin, M. and Balakrishnan, R. 2002. Acquisition of expanding targets. In Proceedings of CHI'O2. ACM, New York, 57-64.

McGuffin, M. and Balakrishnan, R. 2005. Fitts' law and expanding targets: Experimental studies and designs for user interfaces. ACM Trans. Comput.-Hum. Interaction 12, 4, 388-422.

Mead, S. E., Batsakes, P., Fisk, A. D., and Mykityshyn, A. 1999. Application of cognitive theory to training and design solutions for age-related computer use. Int. J. Behav. Develop. 23, 553-573.

Moffatt, K. A. and McGrenere, J. 2007. Slipping and drifting: Using older users to uncover pen-based target acquisition difficulties. In Proceedings of the 9th International ACM SIGACCESS Conference on Computers and Accessibility (Assets'07). ACM, New York, 11-18.

Moffatt, K. A. and McGrenere, J. 2009. Exploring methods to improve pen-based menu selection for younger and older adults. ACM Trans. Access. Comput. 2, 1, Article 3. 1-32.

Moffatt, K. A. and McGrenere, J. 2010. Steadied-bubbles: Combining techniques to address pen-based pointing errors for younger and older adults. In Proceedings of the SIGCHI Conference on Human Factors in Computing Systems (CHI'10). ACM, New York, 1125-1134.

Murata, A. and Iwase, H. 2005. Usability of touch-panel interfaces for older adults. Human Factors: J. Hum. Factors Ergonomics Soc. 47, 4, 767-776.

Nicolau, H. and Jorge, J. 2012. Elderly text-entry performance on touchscreens. In Proceedings of the 14th International ACM SIGACCESS Conference on Computers and Accessibility (ASSETS'12). ACM, New York, 127-134.

Paradise, J., Trewin, S., and Keates, S. 2005. Using pointing devices: difficulties encountered and strategies employed. In Proceedings of the 3rd International Conference on Universal Access in Human-Computer Interaction.

Piper, A. M., Campbell, R., and Hollan, J. D. 2010. Exploring the accessibility and appeal of surface computing for older adult health care support. In Proceedings of the SIGCHI Conference on Human Factors in Computing Systems (CHI'10). ACM, New York, 907-916.

Ruiz, J. and Lank, E. 2010. Speeding pointing in tiled widgets: Understanding the effects of target expansion and misprediction. In Proceedings of the 15th International Conference on Intelligent User Interfaces (IUI'10). ACM, New York, 229-238.

Sandfeld, J. and Jensen, B. R. 2005. Effect of computer mouse gain and visual demand on mouse clicking performance and muscle activation in a young and elderly group of experienced computer users. Appl. Ergonomics 36, 5, 547-555.

Smith, M. W., Sharit, J., and Czaja, S. J. 1999. Aging, motor control, and the performance of computer mouse tasks. Hum. Factors 41, 389-396.

Soukoreff, R. W. and MacKenzie, I. S. 2004. Towards a standard or pointing device evaluation, perspectives on 27 years of Fitts' law research in HCI. Int. J. Hum.-Comput. Stud. 61, 751-789.

Sutter, C. and Müsseler, J. 2007. User specific design of interfaces and interaction techniques: what do older computer users need? In Universal Access in Human Computer Interaction. Coping with Diversity, C. Stephanidis Ed., Lecture Notes in Computer Science, vol. 4554, Springer, Berlin, 1020-1029.

Taveira, A. D. and Choi, S. D. 2009. Review study of computer input devices and older users. Int. J. Hum.Comput. Interaction 25, 5, 455-474.

Trewin, S., Keates, S., and Moffatt, K. 2006. Developing Steady Clicks: A method of cursor assistance for people with motor impairments. In Proceedings of ASSETS'06. ACM, New York, 26-33.

Walker, N., Philbin, D. A., and Fisk, A. D. 1997. Age-related differences in movement control: Adjusting submovement structure to optimize performance. J. Gerontology: Psychol. Sci. 52B, 1, P40-P52.

Wobbrock, J. O., Fogarty, J., Liu, S., Kimuro, S., and Harada, S. 2009. The angle mouse: Target-agnostic dynamic gain adjustment based on angular deviation. In Proceedings of the SIGCHI Conference on Human Factors in Computing Systems (CHI'09). ACM, New York, 1401-1410.

Wood, E., Willoughby, T., Rushing, A., Bechtel, L., and Gilbert, J. 2005. Use of computer input devices by older adults. J. Appl. Gerontology 24, 5, 419-438. 
Worden, A., Walker, N., Bharat, K., and Hudson, S. 1997. Making computers easier for older adults to use: Area cursors and sticky icons. In Proceedings of CHI'97. ACM, New York, 266-271.

Yu, C., Shi, Y., Balakrishnan, R., Meng, X., Suo, Y., Fan, M., and Qin, Y. 2010. The satellite cursor: Achieving MAGIC pointing without gaze tracking using multiple cursors. In Proceedings of the $23 \mathrm{rd}$ Annual ACM Symposium on User Interface Software and Technology (UIST'10). ACM, New York, 163-172.

Zhai, S., Morimoto, C., and Ihde, S. 1999. Manual and gaze input cascaded (MAGIC) pointing. In Proceedings of the SIGCHI Conference on Human Factors in Computing Systems (CHI'99). ACM, New York, 246-253.

Zhai, S., Conversy, S., Beaudouin-Lafon, M., and Guiard, Y. 2003. Human on-line response to target expansion. In Proceedings of CHI'03. ACM, New York, 177-184.

Received September 2012; revised April 2013; accepted June 2013 